\title{
Potential for stem cell-derived biologic pumps for cardiovascular and other medical therapies
}

\author{
Robert A Kloner*,1,2, Sharon L Hale ${ }^{1}$ \& Wangde Dai ${ }^{1,2}$ \\ ${ }^{1}$ Huntington Medical Research Institutes, Pasadena, CA 91105, USA \\ ${ }^{2}$ Keck School of Medicine of University of Southern California, Division of Cardiovascular Medicine and Department of Medicine, \\ University of Southern California, Los Angeles, CA 90033, USA \\ *Author for correspondence: Robert.Kloner@HMRI.org
}

'GFrom these initial studies, we concluded that it is feasible to graft neonatal cardiomyocytes into adult aortas and that these cells survive, differentiate, grow, develop sarcomeres and spontaneously contract."

First draft submitted: 29 April 2019; Accepted for publication: 16 May 2019; Published online: 17 July 2019

Keywords: aorta $\bullet$ biologic pumps $\bullet$ neonatal cardiomyocytes $\bullet$ stem cells $\bullet$ vena cava $\bullet$ ventricular assist

There has been much controversy regarding what, if any, role stem cell therapy will have in the treatment of heart disease, with numerous clinical trials showing that administration of cells into the heart can have discrepant results $[1,2]$. Studies alleging that intrinsic adult cardiac stem cells or bone marrow-derived stem cells can result in transformation to new cardiac muscle have now largely been dismissed by most investigators [3]. Some cells such as CD34 cells and mesenchymal stem cells may promote angiogenesis [4], and CD34 ${ }^{+}$cells have been shown to reduce angina [5] but again, do not create new cardiac muscle. Despite setbacks, the field of stem cell therapy for heart disease is ongoing. There are studies showing that grafts of immature cardiac cells can mature into well-delineated muscle grafts, which can electrically couple with host myocardium and improve cardiac function in myocardial ischemia/infarct models [6-10]. These immature cardiac cells may be derived from neonatal or fetal cardiomyocytes, or from stem cells such as embryonic stem cells or induced pluripotent stem cells. The advantage of the latter is a general lack of an immunologic reaction. However, even this approach is not without difficulty and might be proarrhythmic [10]. In our own experience, if the graft results in cells that have sarcomeres, then the potential for improved contraction is there. If one can create a viable graft of immature cardiac cells that then go on to mature, there is a potential for contraction and improved systolic function of the left ventricle. This concept has largely been applied to implanting cells into diseased hearts as with a myocardial infarction, but the concept can be expanded to include viable biologic pumps that are placed in other parts of the body, besides the hearts.

\section{Testing the biologic pump hypothesis}

In the mid-2000s, our group became interested in the concept of fashioning biologic pumps that could be created by forming a cuff of circumferentially oriented cardiomyocytes around various locations of major blood vessels. We reasoned that such cuffs could survive, form sarcomere-containing cells that contracted, could be paced so that their contraction could eventually be coordinated with contraction of the heart, could generate pressure and eventually contribute to flow. In our first study [11], five million neonatal cardiomyocytes from Fischer rats of either sex or medium (control) were injected under direct vision into the wall of the abdominal aorta of adult female Fischer rats. Of note, we had previously shown that injection of Fischer neonatal cells into adult Fischer rats did not stimulate a rejection phenomenon. The cells were injected into a circumferential cuff totally encircling a segment of the aorta. The rats were allowed to recover for either 2 or 6 weeks. After 2 weeks, the rats were re-anesthetized and the grafted site was examined for the presence of a muscular cuff around the site of the aortic wall injections. In seven of ten cell-treated aortas, spontaneous rhythmic beating at the cuff site was observed after excision of the hearts; no contractions were observed in the control medium injection group. At $3 \mathrm{~min}$ after excision of the hearts, 
the average beating rate of the graft was 102 beats/min. Viable grafts were observed by histology in the aortas of nine out of ten rats at 2 weeks and in six out of six rats at 6 weeks. PCR for the SRY gene (Y chromosome) was positive in the cell-injected aortas but not the medium injected aortas at 6 weeks, confirming that the injected malederived cardiomyocytes were viable in the aortas of adult female rats. Examination using hematoxylin and eosin staining showed well-formed grafts in the treated group with longitudinally (circumferentially oriented) sarcomerecontaining cardiomyocytes encircling the aorta, and a rich neovascularization. At 6 weeks, the cells appeared to be thicker, had more sarcomeres and generally appeared more organized than at 2 weeks. The medium-treated group was devoid of muscle-containing cuffs. The striated cells in the treated group stained positive for the muscle marker sarcomeric actin. From these initial studies, we concluded that it is feasible to graft neonatal cardiomyocytes into adult aortas and that these cells survive, differentiate, grow, develop sarcomeres and spontaneously contract.

\section{Can the grafts generate contractile force?}

In a second study [12], as before, we repeated injections of neonatal cardiomyocytes into the aortas of rats; however, in this study, we assessed the ability of these grafts to be paced and to generate pressure. At 6 weeks after implantation of cells into the outer wall of the abdominal aorta, 11 of 12 aortas demonstrated spontaneously rhythmic beating at the grafted site after excision of the heart. The average rate was 109 beats/min at $1-2 \mathrm{~min}$ after removing the hearts. At 7-8 min after heart excision, the cuff was still beating, although at a slower rate of 53 beats $/ \mathrm{min}$. The cuffs were then paced and responded to pacing (up to $>200$ beats/min). With pacing, the cuffs generated an average pulse pressure of $0.8 \mathrm{mmHg}$; the highest pulse pressure generated was $1.6 \mathrm{mmHg}$ with the systolic pressure up to $3.8 \mathrm{mmHg}$. Histologic analysis revealed compact, circumferentially oriented cardiomyocytes with sarcomeres that stained positive for the muscle marker sarcomeric actin. In addition, the grafted cardiomyocytes stained positive for connexin 43, suggesting that they had formed electrical connections between them. This study showed that neonatal cardiomyocytes grafted into the outer wall of the aorta could not only contract spontaneously but could be paced and generate measurable pressure. The concept with the intra-aortic injections of neonatal cells is that this technique eventually might be used as an auxiliary pump for a weakened left ventricle; a biologic ventricular assist device which becomes a part of the aorta and is used to treat heart failure. To be fully successful, though, a larger number of cells may need to be injected, or multiple injections may need to be performed over time, or it may take $>6$ weeks to develop grafts that can deliver higher pressures. The cuffs would need to be paced in a coordinated fashion with the left ventricle. It is possible that they could be used like an intra-aortic balloon pump, contracting during diastole to improve coronary flow and relaxing during systole to reduce afterload. Or they might be placed in several locations to keep flow moving forward, as in a peristaltic pumping action. So, the ventricle would contract, a proximal aortic cuff pump would initially relax, and then after the blood passes a certain location, it contracts to move blood forward. A second more distally placed cuff might then relax while the first cuff, now contracted, would act as a valve to keep blood flowing forward and not backward. A series of these devices could be used to improve forward flow.

\section{Potential for venous thrombosis?}

It is well known that venous stasis is a major contributor to venous thrombosis and pulmonary embolism. Venous thrombosis typically develops in areas of slow venous flow. Whereas external compression devices have been used, we sought to determine the feasibility of developing a fatigue-resistant pump for venous insufficiency by using the neonatal cardiomyocyte transplantation technique into the walls of veins. In this study [13], five million neonatal cardiomyocytes were injected directly into the wall of the inferior vena cava of adult female Fischer rats. Controls were injected with medium. After 3 weeks, the rats were re-anesthetized and the site of injection was inspected for spontaneously beating. Rhythmic beating of the vena cava was observed at the site of injection in all six rats injected with cells and in none that received medium. The beating rate of the vena cava cuffs was slower (at 92 beats $/ \mathrm{min}$ ) than the aorta. When the heart was excised, the spontaneous beating of the vena cava cuff continued at about the same rate (101 beats/min) at 1-3 min after excision. Histologic assessment revealed viable muscle grafts in all six cell-injected rats and none of the medium injected rats. The morphology was similar to that seen in the intra-aortic grafts, including circumferentially-oriented muscle cells with cross striations, which stained positive for sarcomeric actin. This study showed the feasibility of engrafting neonatal cardiomyocytes around the outer wall of the vena cava and showed that these cells survived in what might have been viewed as a hostile environment, matured, developed contractile units and contracted spontaneously. This technique might someday be further developed to help keep venous flow moving, thereby decreasing venous stasis and venous thromboemboli. 


\section{Promise for the future?}

The same concepts might be applied to other parts of the body. Wherever there is a tube through which fluid flows, biologic pumps could be implanted to help keep flow moving forward. For example, could sluggish gastro-intestinal tracts someday be improved by implanting strategically located biologic pumps derived from immature smooth muscle cells implanted at key locations in the intestines? Could gall stones be eliminated by pumping bile via smooth muscle-generated pumps from the gall bladder to the intestines? Could urinary retention be treated by placing pumps made of smooth muscle cells around key portions of the bladder? Strategic placement of the biologic pumps coupled with pacing technology could conceivably be used for a host of medical conditions. Unfortunately, funding for the development of such technology has been limited, and recent concerns about the controversy surrounding stem cell therapy for the heart will not help. But as long as it has been shown that viable grafts that contain sarcomere-containing cells can be implanted into hostile environments (such as myocardial infarct scars, the walls of aortas and veins) then there is hope for stem cell therapy for the cardiovascular and other systems. While our studies used neonatal cardiomyocytes for cardiovascular applications, there is no reason that similar results would not occur with cardiomyocytes derived from embryonic stem cells or induced pluripotent stem cells. Again, the latter would have an advantage of reduced immunologic response.

\section{Financial \& competing interests disclosure}

The authors have no relevant affiliations or financial involvement with any organization or entity with a financial interest in or financial conflict with the subject matter or materials discussed in the manuscript. This includes employment, consultancies, honoraria, stock ownership or options, expert testimony, grants or patents received or pending, or royalties.

No writing assistance was utilized in the production of this manuscript.

\section{Disclaimer}

The first author certifies that each co-author listed above participated sufficiently in the work to take responsibility for the content, and that all those who qualify are listed.

\section{References}

1. Buja LM. Cardiac repair and the putative role of stem cells. J. Mol. Cell. Cardiol. 128, 96-104 (2019).

2. Wernly B, Mirna M, Rezar R et al. Regerative cardiovascular therapies: stem cells and beyond. Int. J. Mole. Sci. 20(6), 1420 (2019).

3. Vagnozzi RJ, Sargent MA, Lin SJ, Palpant Nj, Murry CE, Molkentin JD. Genetic lineage tracing of Sca-1+cells reveals endothelial but not myogenic contribution to the murine heart. Circulation 138(25), 2931-2939 (2018).

4. Dai W, Hale SL, Martin BJ et al. Allogenic mesenchymal stem cell transplantation in postinfarcted rat myocardium: short-and long-term effects. Circulation 112(2), 214-223 (2005).

5. Henry TD, Losordo DW, Traverse JH et al. Autologous $\mathrm{CD}_{3} 4^{+}$cell therapy improves exercise capacity, angina frequency and reduces mortality in no-option refractory angina: a patient-level pooled analysis of randomized double-blinded trials. Eur. Heart J. 39(23), 2208-2216 (2018).

6. Kadota S, Pabon L, Reinecke H, Murry CE. In vivo maturation of human induced pluripotent stem cell-derived cardiomyocytes in neonatal and adult rat hearts. Stem Cell Reports 8(2), 278-289 (2017).

7. Dai W, Field LJ, Rubart M et al. Survival and maturation of human embryonic stem cell-derived cardiomyocytes in rat hearts. J. Mol. Cell Cardiol. 43(4), 504-516 (2007).

8. Kearns-Jonker M, Dai M, Gunthart M et al. Genetically engineered mesenchymal stem cells influence gene expression in donor cardiomyocytes and the recipient heart. J. Stem Cell Res. Ther. 2012(S1), 005 (2012).

9. Muller-Ehmsen J, Peterson Kl, Kedes L et al. Rebuilding a damaged heart: long-term survival of transplanted neonatal rat cardiomyocytes after myocardial infarction and effect on cardiac function. Circulation 105(14), 1720-1726 (2002).

10. Liu YW, Chen B, Yang X et al. Human embryonic stem cell-derived cardiomyocytes restore function in infarcted hearts of non-human primates. Nat. Biotechnol. 36(7), 597-605 (2018).

11. Dai W, Hale SL, Kloner RA. Implantation of immature neonatal cardiac cells into the wall of the aorta in rats. A novel model for studying morphological and functional development of heart cells in an extracardiac environment. Circulation 110(3), 324-329 (2004).

12. Dai W, Hale SL, Kloner RA. Cardiac cells implanted within the outer aortic call of rats generate measurable contractile force. Regen. Med. 1(1), 119-124 (2006).

13. Dai W, Hale SL, Kloner RA. Development of a spontaneous beating vein by cardiomyocyte transplantation in the wall of the inferior vena cava in a rat: a pilot study. J. Vasc. Surg. 45(4), 817-820 (2007). 